\title{
Closure of the ductus arteriosus and development of pulmonary branch stenosis in babies of less than 32 weeks gestation
}

\author{
R Arlettaz, N Archer, A R Wilkinson
}

\begin{abstract}
Aims-To define how often transient pulmonary branch stenosis (PBS) develops after closure of a patent ductus arteriosus (PDA) in babies born at less than 32 weeks gestation; to describe the natural history of PBS and the relation between PBS and a cardiac murmur.

Methods-Fifty three preterm infants born at a gestational age less than 32 weeks and who had PDA diagnosed on echocardiography were recruited. An echocardiogram was performed on alternate days until the ductus arteriosus closed. If PBS was diagnosed, the baby was followed up until PBS resolved.

Results-In 59\%, PBS developed in one or both branches after closure of the PDA. In $21 \%$, both pulmonary branches were affected. In $79 \%$, the left pulmonary artery alone was involved but the right side was never affected alone. PBS had resolved in $74 \%$ by the time the infants reached 40 weeks, in $95 \%$ at a corrected age of 6 weeks, and in $100 \%$ at a corrected age of 3 months. There is a better correlation between a cardiac murmur and PBS than between a murmur and PDA.

Conclusions-PBS in preterm infants is usually not present at birth but develops after closure of a PDA. PBS resolves by a corrected age of 3 months. The presence of a murmur after closure of a PDA is usually related to PBS and not to reopening of the ductus arteriosus.
\end{abstract}

(Arch Dis Child Fetal Neonatal Ed 2001;85:F197-F200)

Keywords: pulmonary branch stenosis; patent ductus arteriosus; cardiac murmur; preterm infants

Transient pulmonary branch stenosis (PBS) in preterm and term neonates is well recognised. ${ }^{1-3}$ In preterm infants, closure of a patent ductus arteriosus (PDA) seems to be an important factor in the development of PBS, ${ }^{45}$ but in only one of these studies was the natural history reported. The presence of a heart murmur a few days or weeks after closure of a PDA in a preterm infant is often thought to be due to reopening of the ductus arteriosus, but the relation between a murmur and PDA is poor, ${ }^{6-10}$ and in term infants it has been shown to be more often associated with PBS than with PDA. ${ }^{11}$

The aims of this study were to (a) describe how often PBS develops in preterm infants after closure of the ductus arteriosus, (b) describe the natural history of PBS in preterm infants, and (c) determine the relation between PBS and the presence of a murmur.

\section{Methods}

Preterm infants of gestational age below 32 weeks had a full clinical examination performed by the resident paediatrician and then a complete echocardiogram performed between the second and sixth day after birth. Infants with a PDA were recruited for the study, but those with congenital heart disease were excluded. A selective echocardiogram, to study the velocity pattern across the main pulmonary artery (MPA), the right and left pulmonary arteries (RPA and LPA), as well as the size and velocity pattern across the ductus arteriosus, was then carried out on alternate days until the ductus arteriosus closed. Two further studies were carried out during the week after ductal closure. Infants who developed PBS had further selective echocardiograms performed every two weeks until discharge from hospital. If PBS was still present at discharge, the baby was followed up until PBS had resolved completely by repeat studies at a corrected age of 6 weeks, 3 months, and 6 months. All echocardiograms were performed by the same investigator (RA).

The ultrasound studies were carried out with an Acuson 128XP/10 displaying simultaneous electrocardiograms and equipped with all Doppler modalities. A gap in the atrial septum of $3 \mathrm{~mm}$ diameter or less was not considered to be a structural heart disease. PBS was defined as an acceleration of the peak flow velocity of at least $50 \%$ in one or both pulmonary artery branches compared with the MPA. ${ }^{4}$ The pulmonary valve, the MPA, and its branches were visualised on the parasternal short axis view. The internal diameter of the pulmonary valve was measured at its maximal opening, and the diameters of the right and left pulmonary arteries were measured at their origin during systole. For pulmonary Doppler assessment, the pulsed Doppler sample volume was placed distal to the pulmonary valve, and the pulmonary artery blood velocity was measured. The pulsed Doppler sample volume was then moved to the origin of the RPA and LPA, and the velocity was recorded. The Doppler assessment of the LPA was recorded from the high parasternal view in addition, and the greater velocity was used in the analysis. The ductus arteriosus was visualised in the high parasternal view, and the velocity pattern was noted.

The babies were not sedated for the examinations. The study was approved by the 
Table 1 Doppler measurements in infants with and without patent ductus arteriosus

\begin{tabular}{|c|c|c|c|c|c|}
\hline & \multicolumn{2}{|c|}{$\begin{array}{l}\text { Ductus arteriosus open } \\
(n=79)\end{array}$} & \multicolumn{2}{|c|}{$\begin{array}{l}\text { Ductus arteriosus closed } \\
(n=253)\end{array}$} & \multirow[b]{2}{*}{$p$ Value } \\
\hline & Median & Range & Median & Range & \\
\hline \multicolumn{6}{|c|}{ Peak velocities $(\mathrm{cm} / \mathrm{s})$} \\
\hline MPA & 63 & $46-95$ & 79 & $46-133$ & 0.0001 \\
\hline RPA & 71 & $44-118$ & 97 & $49-173$ & 0.0001 \\
\hline LPA & 80 & $41-175$ & 127 & $71-240$ & 0.0001 \\
\hline \multicolumn{6}{|l|}{ Acceleration (\%) } \\
\hline MPA to RPA & 11 & $0-72$ & 22 & $1-179$ & 0.0001 \\
\hline MPA to LPA & 18 & $0-113$ & 63 & $2-247$ & 0.0001 \\
\hline
\end{tabular}

MPA, Main pulmonary artery; RPA, right pulmonary artery; LPA, left pulmonary artery.

Table 2 Doppler measurements in infants without pulmonary branch stenosis (PBS), with $P B S$ on the left, and with PBS on the left and right after closure of the ductus arteriosus

\begin{tabular}{llllll}
\hline & $\begin{array}{l}\text { No PBS } \\
(n=80)\end{array}$ & $\begin{array}{l}\text { PBS left } \\
(n=100)\end{array}$ & $p$ Value & $\begin{array}{l}\text { PBS left and right } \\
(n=26)\end{array}$ & $p$ Value \\
\hline $\begin{array}{l}\text { Peak velocities }(\mathrm{cm} / \mathrm{s}) \\
\text { MPA }\end{array}$ & $80(52-123)$ & $76(46-133)$ & 0.79 & $77(53-101)$ & 0.09 \\
RPA & $91(49-143)$ & $94(61-165)$ & 0.13 & $119(85-173)$ & 0.0001 \\
LPA & $110(71-213)$ & $132(71-217)$ & 0.0002 & $157(2-220)$ & 0.0001 \\
$\begin{array}{l}\text { Acceleration (\%) } \\
\text { MPA to RPA }\end{array}$ & $12(0-81)$ & $22(0-136)$ & 0.02 & $60(1-179)$ & 0.0001 \\
MPA to LPA & $36(0-156)$ & $69(0-178)$ & 0.0001 & $121(2-247)$ & 0.0001 \\
\hline
\end{tabular}

Values are median (range)

MPA, Main pulmonary artery; RPA, right pulmonary artery; LPA, left pulmonary artery.

Table 3 Comparison of the incidence of pulmonary branch stenosis (PBS) in indomethacin treated compared with spontaneous closed patent ductus arteriosus (PDA)

\begin{tabular}{llll}
\hline & $\begin{array}{l}P D A \text { closed with } \\
\text { indomethacin }(n=22)\end{array}$ & $\begin{array}{l}P D A \text { closed } \\
\text { spontaneously }(n=29)\end{array}$ & $p$ Value \\
\hline Gestational age (weeks) & $28(25-30)$ & $30(24-31)$ & 0.0001 \\
Birth weight (g) & $942(670-1320)$ & $1255(700-1870)$ & 0.0002 \\
Incidence of PBS (\%) & $69(11 / 16)^{\star}$ & $52(13 / 52) \dagger$ & 0.13 \\
\hline
\end{tabular}

The results are expressed as median (range).

${ }^{\star}$ Six infants already had PBS at birth.

†Four infants already had PBS at birth.

local hospital research ethics committee, and informed consent from one or both parents was obtained in all cases.

Statistical analyses were performed using $t$ tests and $\chi^{2}$ test. $\mathrm{p}<0.05$ was considered to be significant.

\section{Results}

During 14 months, 83 babies out of 95 who were eligible were recruited. Thirty were excluded because the ductus arteriosus was already closed at the time of the first echocardiogram. Fifty three babies (24 boys and 29 girls) were therefore included in the study. The babies had a median gestational age of 28 weeks (range 24-31) and a median birth weight of $1060 \mathrm{~g}$ (range 670-1870). Valvular pulmonary stenosis was diagnosed at 3 and 8 weeks of age in two babies who were excluded from the final analysis. The remaining 51 infants form the basis of this report.

Respiratory distress (defined as the presence of at least two of the five following clinical signs: tachypnoea $>60 / \mathrm{min}$, grunting, subcostal or intercostal retractions, nasal flaring, $\mathrm{SaO}_{2}<85 \%$ while breathing air) was diagnosed in 49 infants $(96 \%)$. Five infants had respiratory distress of unknown origin while the remaining had either hyaline membrane disease or transient tachypnoea. One third of the study infants (18/51) received mechanical ventilation, followed in most by nasal continuous positive airway pressure; nearly half (24/51) required nasal continuous positive airway pressure only. Fourteen infants $(27 \%)$ with a median gestational age of 27 weeks required supplemental oxygen at a corrected age of 36 weeks. Four babies $(7.5 \%)$ died between days 10 and 23. The remaining infants were discharged from hospital at a median corrected age of 38 weeks gestation (range 35-43).

A total of 346 echocardiograms were performed, a median of seven echocardiograms per infant (range 2-11).

\section{PATENT DUCTUS ARTERIOSUS}

The first echocardiogram was performed between day 2 and 6 . This first study showed a structurally normal heart in all infants and a PDA. Symptomatic PDA was treated according to contemporary recommendations. ${ }^{12}$ Twenty two of the 51 infants (43\%) were given a course of treatment with indomethacin, and the ductus arteriosus closed in all except two infants who had a PDA until discharge. In the remaining 29 infants, the ductus arteriosus closed spontaneously. No infant required surgical ligation of the PDA. The ductus arteriosus closed at a median postnatal age of five days (range 3-15).

PULMONARY ARTERY DOPPLER MEASUREMENTS WITH DUCTUS ARTERIOSUS OPEN

The 51 infants had a total of 79 echocardiograms in which the ductus arteriosus was patent. Table 1 shows the measurements of the peak velocities across the pulmonary valve, the RPA, and the LPA, as well as the acceleration between the MPA and the branches.

PULMONARY ARTERY DOPPLER MEASUREMENTS WITH DUCTUS ARTERIOSUS CLOSED

The 51 infants had a total of 253 echocardiograms in which the ductus arteriosus was closed (table 1). These velocities and accelerations are higher than those observed with a PDA $(\mathrm{p}<0.0001)$.

DEVELOPMENT OF PBS AFTER CLOSURE OF THE PDA

Of the 51 infants, 10 were not considered for the development of PBS because eight already had PBS when the ductus arteriosus was patent and two had a PDA until discharge. Of the remaining 41 infants, 24 (59\%) developed PBS. In nineteen this was in the LPA, in five on both right and left sides, but in none on the right side alone (table 2 ).

The Doppler measurements in these three groups showed that the Doppler peak velocity in the MPA was not statistically different between groups.

We compared the incidence of PBS in infants given indomethacin $(n=22)$ with those with spontaneous ductal closure $(n=29)$. There was no significant difference in incidence of PBS between the groups (table 3).

PRESENCE OF PBS FROM BIRTH

Eight of the 51 infants (15\%) showed PBS on the first echocardiogram along with PDA. The incidence of PBS with PDA is therefore much lower than after ductal closure (59\%). This 
Table 4 Follow up of infants who developed pulmonary branch stenosis (PBS) compared with those with PBS present at birth

\begin{tabular}{llll}
\hline & $\begin{array}{l}\text { Infants who } \\
\text { developed PBS } \\
(n=19)\end{array}$ & $\begin{array}{l}\text { Infants with PBS } \\
\text { present at birth } \\
(n=5)\end{array}$ & p Value \\
\hline $\begin{array}{l}\text { Gestational age (weeks) } \\
\begin{array}{l}\text { Birth weight (g) } \\
\text { PBS resolved }\end{array}\end{array}$ & $28(24-31)$ & $28(26-29)$ & \\
$\quad \begin{array}{l}\text { Days of life } \\
\text { Corrected gestational age (weeks) }\end{array}$ & $58(680-1870)$ & $1090(840-1540)$ & \\
\hline
\end{tabular}

The results are expressed as median (range).

difference is highly significant $(\mathrm{p}<0.001)$. These eight infants had a gestational age and birth weight that did not differ statistically from the rest of the group. In all but one infant, the severity of PBS increased after closure of the ductus arteriosus.

RELIABILITY OF A MURMUR IN THE PRESENCE OF PBS

The correlation between a systolic murmur and the presence of PBS was tested in all measurements with the ductus arteriosus closed $(n=253)$. In 147 scans, PBS was detected; on 34 occasions a murmur was heard. In 106 scans no PBS was detected, and on 14 occasions the baby had a murmur. The sensitivity of a murmur for PBS is therefore $23 \%$ and the specificity $87 \%$. If PBS is defined as a Doppler peak velocity above $150 \mathrm{~cm} / \mathrm{s}$, the sensitivity of a murmur to detect PBS is $40 \%$ and the specificity $88 \%$.

FOLLOW UP

Of the 24 babies who developed stenosis of one or both pulmonary artery branches, two died and three were transferred to other hospitals. The remaining 19 babies were followed up until PBS resolved. PBS resolved at a median postnatal age of 53 days (range 10-144); the median gestational age was 36 corrected weeks (range 29-52).

In five of 19 infants (26\%), PBS was still detected at 40 weeks and resolved at a corrected age of 6 weeks in four and three months in the last infant. Therefore PBS resolved in $74 \%$ infants (14/19) by 40 weeks, in $95 \%(18 / 19)$ at 6 corrected weeks, and in $100 \%$ at 3 corrected months. PBS resolved earlier in the group of infants who developed PBS ( $n=19)$ than in those who had PBS at birth $(n=5)$. This difference is significant (table 4$)$.

\section{Discussion}

PBS can be easily detected by echocardiography. There is a discrepancy between the size of the MPA and that of the right and left branches; the velocity at the origin of the branches is significantly higher than that measured in the pulmonary trunk, and the flow at the origin of the branches is turbulent on colour flow Doppler. The standard definition for PBS used by most investigators is that of Maroto et $a l,{ }^{4}$ namely an increase at the origin of the RPA or LPA branch in the peak flow velocity of at least $50 \%$ when compared with the value recorded in the MPA.

PBS in a neonate is usually physiological and transient. The pressure difference between the MPA and one or both branches rarely exceeds
$15 \mathrm{~mm} \mathrm{Hg},{ }^{1}$ so that the repercussion on the pulmonary circulation is minimal. The PBS is transient and resolves when the branches of the pulmonary artery have enlarged. This physiological PBS is to be differentiated from the pathological peripheral pulmonary stenosis associated with congenital heart disease such as in Allagille syndrome or congenital rubella, in which the pulmonary branches show a fixed structural stenosis.

Several authors 2351314 mention a higher incidence of PBS in preterm than in term neonates. They speculate that PBS is related to the diameter of the pulmonary artery branches which is smaller in immature infants.

Further, the systolic murmur associated with PBS develops later after birth in preterm than in term infants. ${ }^{513}$ This could be explained by the fact that the pressure difference between the pulmonary artery and the branches increases when the pulmonary pressure decreases, which occurs later in preterm infants. ${ }^{15}$ The delayed closure of the ductus arteriosus results in an increase in ventricular output with an increase in velocity in the MPA and both branches.

This study confirms the results of Maroto et $a{ }^{4}{ }^{4}$ PBS developed in more than half of the babies after closure of the ductus arteriosus. A stenosis of the LPA is much more common than that of the right branch. This phenomenon has been described by other authors ${ }^{314} 16$ and can be explained by two hypotheses. The first one is a technical difficulty with echocardiography. The angle between the RPA and the Doppler cursor can possibly underestimate the true value of the Doppler velocities across the RPA. This is not the case for the LPA which arises parallel to the Doppler cursor on the high parasternal view and thus can be easily measured. The second hypothesis arises from histological studies of Gittenberger-de Groot et $a l .{ }^{17}{ }^{18}$ and Zevallas-Giampietri. ${ }^{19}$ They showed that the tissue at the transition between aorta and ductus arteriosus, called the roof of the ductus arteriosus, is regular and progressive. In contrast, at the pulmonary end, the ductus arteriosus merges abruptly with the elastic wall at the bifurcation between the pulmonary trunk and the branches, particularly the left one, which is in contact with more ductal tissue. The constriction of the ductus arteriosus, with its fibres of smooth muscle arranged spirally, causes not only a narrowing but also a shortening, ${ }^{1}$ which itself causes traction that is more pronounced at the origin of the left pulmonary branch. This traction emphasises the angle between the trunk and the branches and is responsible for the acceleration of the blood and for the presence of turbulence. ${ }^{2} 1416$ This phenomenon has been described as "juxtaductal pulmonary artery coarctation". A similar explanation has been proposed for the postnatal appearance of coarctation of the aorta. ${ }^{320}$

In this study, eight (15\%) preterm infants had PBS present at birth when the ductus arteriosus was still patent. This is not surprising because after birth the pulmonary blood flow increases dramatically because of the decrease 
in pulmonary vascular resistance. The increasing flow across the pulmonary trunk and its branches emphasises the pressure difference. It would also be expected that with a large left to right shunt across the ductus arteriosus or the foramen ovale, the pressure difference between the trunk and the branches would be even greater. Danilowitz et al showed this to be the case in a study confirmed by cardiac catheterisation. Maroto et $a l,{ }^{4}$ however, did not observe any PBS with PDA.

The results of this study show a poor correlation between the presence of a murmur and PBS using the definition of Maroto et al. ${ }^{4}$ This is not surprising as Maroto used an increase in velocity as a definition criterion, which is not associated with a murmur. A murmur is caused by a pressure gradient that is usually observed when the Doppler peak velocity exceeds 150 $\mathrm{cm}$ per second. According to this clinical definition, the sensitivity of the murmur to detect PBS is $40 \%$ and the specificity $88 \%$. For Doppler velocities above $200 \mathrm{~cm}$ per second, a murmur was heard in $77 \%(10 / 13)$ of babies. This is much better than the correlation between a murmur and PDA, which is 14-28\%..$^{6-10}$ Few studies have evaluated the reliability of a murmur in the presence of PBS. In most of them, ${ }^{511}{ }^{14}$ the babies were recruited because of a murmur and not because of PBS. Further, PBS is defined using various criteria, which makes a comparison impossible.

Few studies have followed up babies with PBS. One reported that, in 14 infants, PBS had resolved in $64 \%$ at 3 months of age and in all of them by the age of 9 months. ${ }^{14}$ In another study of 25 low birthweight infants, the resolution of PBS and the murmur was observed by $2-5$ months of age. ${ }^{5}$ A further study describes the resolution of PBS within 3-6 months in $40 \%$ of 30 term babies. ${ }^{21}$ Our previous experience of 44 term infants showed resolution of PBS in $88 \%$ at 3 months of age and in all of them by the age of 6 months. ${ }^{11}$ The current data support the results of these previous studies.

We conclude that PBS in preterm infants is usually not present at birth but develops after closure of PDA. PBS resolves in three quarters by a corrected age of 40 weeks. The presence of a murmur after closure of PDA is usually related to PBS and not to reopening of the ductus arteriosus.

There are two clinical implications of this study: firstly, preterm infants who have had a PDA, treated or not with indomethacin, can present with a systolic murmur a few days or up to six weeks after closure of the ductus arteriosus. The murmur, which is often thought to be due to reopening of the ductus arteriosus, is more likely to be caused by PBS. Secondly, PBS is physiological and resolves by a corrected age of 3 months in all infants.

1 Rudolph AM. The pre- and postnatal pulmonary circulation. In: Congenital diseases of the heart. Chicago: Year Book Medical Publishers, 1974:29-37;168-72.

2 Danilowicz DA, Rudolph AM, Hoffman JIE, et al. Physiologic pressure differences between main and branch Physiologic pressure differences between main and branch
pulmonary arteries in infants. Circulation 1972;45:410-19. pulmonary arteries in infants. Circulation $1972 ; 45: 410-19$.
3 Rodriguez RJ, Riggs TW. Physiologic peripheral pulmonic stenosis in infancy. Am $\mathcal{F}$ Cardiol 1990;66:1478-81.

4 Maroto E, Fouron JC, Aké E, et al. Closure of the ductus arteriosus: determinant factor in the appearance of transient peripheral pulmonary stenosis of the neonate. $\mathcal{F}$ Pediatr 1991;119:955-9.

5 So BH, Watanabe T, Shimizu M, et al. Doppler assessment of physiological stenosis at the bifurcation of the main pulmonary artery: a cause of functional murmur in neonates. Biol Neonate 1996;69:243-8.

6 Braudo M, Rowe RD. Auscultation of the heart: early neonatal period. American fournal of Diseases in Children 1961;101:575-86

7 Gentile R, Stevenson G, Dooley T, et al. Pulsed Doppler echocardiographic determination of time of ductal closure in normal newborn infants. 7 Pediatr 1981:98:443-8.

8 Reller MD, Colasurdo MA, Rice MJ, et al. The timing of spontaneous closure of the ductus arteriosus in infants with respiratory distress syndrome. Am $\mathcal{f}$ Cardiol 1990;66:75-8.

9 Freund $M$ and Wranne B. Ultrasound assessment of ductal closure, pulmonary blood flow velocity, and systolic pulmonary arterial pressure in healthy neonates. Pediatr Cardiol 1986;6:233-7.

10 Thibeault DW, Emmanouilides GC, Nelson RJ, et al. Patent ductus arteriosus complicating the respiratory distress syndrome in preterm infants. F Pediatr 1975;86:120-6.

11 Arlettaz R, Archer N, Wilkinson AR. Natural history of innocent heart murmurs in newborn babies: controlled echocardiographic study. Arch Dis Child Fetal Neonatal Ed 1998;78:F166-70.

12 Clyman R. Recommendations for the postnatal use of indomethacin: an analysis of four separate treatment strategies. F Pediatr 1996;128:601-7.

13 Dunkle LM, Rowe RD. Transient murmur simulating pulmonary artery stenosis in premature infants. American pulmonary artery stenosis in premature infants.
fournal of Diseases in Children 1972;124:666-70.

14 Chatelain P, Oberhänsli I, Friedli B. Physiological pulmonary branch stenosis in newborns: 2D-echocardiographic and Doppler characteristics and follow up. Eur $\mathcal{F}$ Pediatr 1993;152:559-63

15 Evans NJ, Archer NJ. Postnatal circulatory adaptation in healthy term and preterm neonates. Arch Dis Child 1990;65:24-6.

16 Gunteroth WG. The role of ductal constriction in transient stenosis of the left pulmonary artery. Pediatr Cardiol 1998;19:240-2l.

17 Gittenberger-de Groot AC, Strengers JLM, Mentink M, et al. Histologic studies on normal and persistent ductus arteriosus in the dog. 7 Am Coll Cardiol 1985;6:394-404.

18 Gittenberger-de Groot AC, van Ertbruggen I, Moulaert A, et al. The ductus arteriosus in the preterm infant: histologic and clinical observations. $\mathcal{F}$ Pediatr 1980;96:8893.

19 Zevallos-Giampietri EA, Thelmo WL, Anderson VM. Coarctation of the left pulmonary artery: effects on the pulmonary vasculature of infants. Pediatr Cardiol 1997;100:376-80.

20 Elzenga NJ, van Suylen RJ, Frohn-Mulder I, et al. Juxtaductal pulmonary artery coarctation. $\mathcal{F}$ Thorac Cardiovasc Surg 1990;18:416-24.

21 Du ZD, Roguin N, Barak M, et al. Doppler echocardiographic study of the pulmonary artery and its branches in 114 normal neonates. Pediatr Cardiol 1997;18:38-42. 